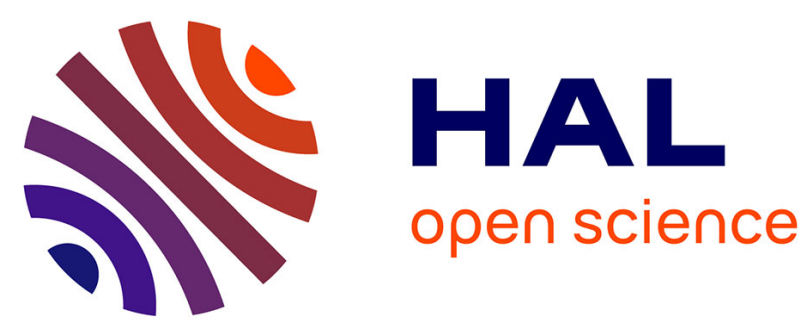

\title{
Sex-specific spatial variation in fitness in the highly dimorphic Leucadendron rubrum
}

Jeanne Tonnabel, Etienne K. Klein, Ophélie Ronce, Sylvie Oddou-muratorio, François Rousset, Isabelle Olivieri, Alexandre Courtiol, Agnès Mignot

\section{- To cite this version:}

Jeanne Tonnabel, Etienne K. Klein, Ophélie Ronce, Sylvie Oddou-muratorio, François Rousset, et al.. Sex-specific spatial variation in fitness in the highly dimorphic Leucadendron rubrum. Molecular Ecology, 2021, 30 (7), pp.1721-1735. 10.1111/mec.15833 . hal-03279442

HAL Id: hal-03279442

https: / hal.inrae.fr/hal-03279442

Submitted on 25 Nov 2021

HAL is a multi-disciplinary open access archive for the deposit and dissemination of scientific research documents, whether they are published or not. The documents may come from teaching and research institutions in France or abroad, or from public or private research centers.
L'archive ouverte pluridisciplinaire HAL, est destinée au dépôt et à la diffusion de documents scientifiques de niveau recherche, publiés ou non, émanant des établissements d'enseignement et de recherche français ou étrangers, des laboratoires publics ou privés.

\section{(c)(1)}

Distributed under a Creative Commons Attribution| 4.0 International License 
3 Running title: Sex-specific spatial variation in fitness

4 Jeanne Tonnabel ${ }^{1,2, * \S}$, Etienne K. Klein ${ }^{3}$, Ophélie Ronce ${ }^{1,4}$, Sylvie Oddou-Muratorio ${ }^{5}$, François

5 Rousset $^{1}$, Isabelle Olivieri ${ }^{1}$, Alexandre Courtiol ${ }^{61}$, Agnès Mignot ${ }^{1 \mathrm{f}}$

$6 \quad{ }^{1}$ ISEM, Université de Montpellier, CNRS, IRD, EPHE Montpellier, France.

$7 \quad{ }^{2}$ Department of Ecology and Evolution, Le Biophore, UNIL-SORGE, University of Lausanne, CH81015 Lausanne, Switzerland.

$9{ }^{3}$ INRAE, BioSP, F-84000, Avignon, France.

$10 \quad{ }^{4}$ CNRS, Biodiversity Research Center, University of British Columbia, Vancouver, Canada

$11{ }^{5}$ INRAE, URFM, F-84000, Avignon, France.

$12{ }^{6}$ Department of Evolutionary Genetics, Leibniz Institute for Zoo and Wildlife Research, 10315

13 Berlin, Germany.

$14{ }^{\mathrm{I}}$ Co-senior authors

$15 \quad{ }^{*}$ Corresponding author: jeanne.tonnabel@cefe.cnrs.fr

$16 \S$ Current address: Centre d'Ecologie Fonctionnelle et Evolutive (CEFE), UMR 5175, CNRS, UM,

17 Université Paul Valéry Montpellier, EPHE, 1919 route de Mende, 34293 Montpellier Cedex 5, 18 France. 


\section{Abstract}

Sexual dimorphism in plants may emerge as a result of sex-specific selection on traits enhancing access to nutritive resources and/or to sexual partners. We here investigated sexspecific differences in selection of sexually dimorphic traits and in the spatial distribution of effective fecundity (our fitness proxy) in a highly dimorphic dioecious wind-pollinated shrub, Leucadendron rubrum. In particular, we tested for the effect of density on male and female effective fecundity. We used spatial and genotypic data of parent and offspring cohorts to jointly estimate individual male and female effective fecundity on the one hand and pollen and seed dispersal kernels on the other hand. This methodology was here adapted to the case of dioecious species. Explicitly modeling dispersal avoids the confounding effects of heterogeneous spatial distribution of mates and sampled seedlings on the estimation of effective fecundity. We also estimated selection gradients on plant traits while modeling sex-specific spatial autocorrelation in fecundity. Males exhibited spatial autocorrelation in effective fecundity at a smaller scale than females. A higher local density of plants was associated with lower effective fecundity in males but was not related to female effective fecundity. These results suggest sex-specific sensitivities to environmental heterogeneity in L. rubrum. Despite these sexual differences, we found directional selection for wider canopies and smaller leaves in both sexes, and no sexually antagonistic selection on strongly dimorphic traits in L. rubrum. Many empirical studies in animals similarly failed to detect sexually antagonistic selection in species expressing strong sexual dimorphism, and we discuss reasons explaining this common pattern.

Keywords: sexual dimorphism, sexual selection, selection gradients, dispersal kernels, cost of reproduction, spatial structure 


\section{Introduction}

Plant species with separate sexes are relatively uncommon (i.e. 5-6\%, Renner, 2014). Separate sexes have nonetheless evolved repeatedly among flowering plants (Renner, 2014), and such transitions 
needed for their respective reproduction. The cost of reproduction is generally higher in females

than in males, at least considering the cost per reproductive structure. However, at the scale of the whole plant, this trend is often reversed in wind-pollinated plants, which produce large amounts of pollen (Obeso, 2002; Harris \& Pannell, 2008; Tonnabel et al., 2017). In some dioecious species inhabiting fire-prone environments, the cost of reproduction markedly differs between sexes because females need to maintain a canopy-stored ('serotinous') seed bank (released by fire). As water intake is necessary to maintain cones closed and prevent seed release during unfavorable period between two fires (Martín-Sanz et al., 2017), we may expect selection for enhanced water conduction to have favored a divergent plant architecture between sexes. Consistent with this prediction, the evolution of longer maintenance of cones in the canopy is indeed associated with the evolution of higher sexual dimorphism in the genus Leucadrendron (Harris \& Pannell, 2010).

The evolution of sex-specific differences in vegetative traits may also originate from selection for male morphologies that are better at dispersing pollen and therefore at accessing mates (Tonnabel et al., 2019a,b). The male-male competition hypothesis postulates that male reproduction, by being mostly limited by mating opportunities, selects for males that exhibit traits enhancing their competitive abilities (Bateman, 1948; Arnold, 1994). Several studies have pinpointed the importance of male-male competition in shaping male reproductive and floral traits. These studies showed more extravagant floral displays in males than in females to attract pollinators (e.g. Bond \& Maze, 1999; Elle \& Meagher, 2000; Wright \& Meagher, 2004; Delph \& Ashman, 2006; Waelti et al., 2009; Schiestl \& Johnson, 2013; Dorken \& Perry, 2017), variation in male flowering phenology to track the female phenology (Delph \& Herlihy, 2011; Forrest, 2014), increased pollen grain competitive performance in response to polyandry (Lankinen et al., 2017) and morphological evolution of structures that disperse pollen, which prevents the attachment of competing pollen to the pollinator (Coccuci et al., 2014). In wind-pollinated plants, sexual selection may also target vegetative traits such as plant size, branch length or the length of male flower peduncles, which can facilitate pollen dispersal (Klinkhamer et al., 1997; Eppley \& Pannell, 2007; Pickup \& Barrett, 
94

2012; Harder \& Prusinkiewicz, 2013; Tonnabel et al., 2019b). Wind-pollinated plants tend to evolve larger degrees of sexual dimorphism than insect-pollinated lineages, because pollinators require similarity of floral morphology to successfully transfer pollen (Tonnabel et al., 2014; Welsford et al., 2016). Wind-pollinated plants, which typically show large inter-individual variation in fertility (Schoen \& Stewart, 1987; Ahee et al., 2015), may thus be particularly subject to sexually antagonistic selection.

In the presence of genetic variation for sexual dimorphism, each sex should, in principle, ultimately reach its optimal trait value, and thereby resolve sexually antagonistic selection (Lande, 1980). Yet, a shared genetic basis of traits between sexes may temporally constrain the evolution of their morphological divergence (Lande, 1980). In constant and homogeneous environments, theory predicts that, with strong positive genetic correlations between sexes, opposite directional selection gradients between sexes should emerge early during adaptation and persist for a long time before the sexual conflict is resolved (Lande, 1980; Connallon \& Hall, 2016). Consequently, one would expect evidence for antagonistic selection between sexes to be relatively common. The compilation of numerous sex-specific selection gradients in animals showed, however, a large diversity of patterns, including cases of aligned selection across sexes (Cox \& Calsbeek, 2009). In plants, documented patterns of sex-specific selection provided mixed support for sexually antagonistic selection: sexspecific selection gradients have been found to have opposite signs in both insect- and windpollinated species (Delph et al., 2011; Castilla et al., 2014; Tonnabel et al., 2019b) but to have the same sign in other studies (Oddou-Muratorio et al., 2018; Barrio \& Teixido, 2014). More recent theory suggests that temporal and spatial variation in selection pressures may explain the lack of signal for sexually antagonistic selection, despite differences in the optimal phenotypes of males and females (Connallon, 2015; Connallon \& Hall, 2016; Zafitscher \& Connallon, 2017).

Estimating sex-specific selection gradients requires, first, estimating male and female individual fitness, and, second, relating trait values and fitness estimates. Using genotypes of established seedlings and their potential parents, traditional methods first achieve categorical 
parentage assignments to then estimate individual realized reproductive successes used as fitness

estimates. In the next generation methods, genotypes are combined with spatial localization of sampled individuals, through spatially explicit mating models (SEMMs), to disentangle the effect of fecundity from that of the distance between mating pairs (and the distance between mothers and seedlings) on reproductive success (e.g. Oddou-Muratorio et al., 2005). To do so, dispersal is explicitly modeled and dispersal kernels are estimated for both seeds and pollen. A Bayesian method was introduced in this framework to estimate individual male and female effective fecundities (MEMM, Klein et al., 2008 for seed sampling on mother trees; MEMMseedlings, Oddou-Muratorio et al., 2018 for seedling sampling designs). This method considers the likelihood of genotypes conditional on the position of seedlings, so it is unaffected by any process acting on the distribution of seedlings, be it the potential parents' positions, or habitat suitability and disturbances. It analyses seedling genotypes in terms of (1) overall reproductive contribution of each potential parent as determined jointly by gamete production, gamete fertilization rates, seed maturation and germination, and seedling survival until census; (2) dispersal events in terms of estimated dispersal kernels and (3) pollen or seedling competition by a mass action law. Effective fecundity refers only to relative values of the first component for each parent. It varies with, e.g., male-male competitive ability through differences in overall pollen production and their subsequent ovule fertilization abilities, but not with competitive effects dependent on the composition of competitors within the pollen cloud generated by uneven spatial distribution of mates. We here extend this methodology to dioecious species. This spatially explicit approach avoids spatial bias in effective fecundity estimation, typically generated by sampling seedlings non-uniformly with respect to the positions of their parents or by the confounding effects of heterogeneous spatial distribution of mates (Oddou-Muratorio et al., 2018). Used as a proxy for fitness, effective fecundity thus provides the expected relative reproductive success if putative mates (for male fecundity) and regeneration sites (for female fecundity) were uniformly distributed in space, and all offspring could 
establish and be sampled (Klein et al., 2013). It therefore attenuates the impact of stochastic effects associated with sampling methods on fitness estimates.

Relating fitness estimates to plant traits using the selection gradients methodology proposed

by Lande \& Arnold (1983) can further suffer from specific statistical bias in sessile organisms living in heterogeneous environments. Indeed, small-scale spatial variation in resources fundamental to plant physiology, including sex-specific reproduction, is common across a range of habitat types (Silvertown et al., 1999; Araya et al., 2011). To disentangle the fitness effect of plant characteristics (such as their ability to harvest resources, which may be sex-specific) from that of the environment (such as the spatial distribution of resources), the spatial distribution of individuals must be accounted for (Rausher, 1992). Indeed, not modeling explicitly the spatial autocorrelation of unmeasured ecological variables affecting fitness can lead to detect false-positive effects of traits on fitness, as on any other response (Guillot \& Rousset, 2013). To address this problem, we fitted mixed-effect models with spatially autocorrelated random-effects, using the spaMM package (Rousset \& Ferdy, 2014). To our knowledge, it is the first time that spatial effects are taken into account in the estimation of selection gradients. Moreover, spatial variation in plant density and the local sex ratio may generate spatial variation in competition for resources, which can be studied by analyzing their fixed effects on plant fitness. In conclusion, our MEMMseedlings model controls for spatial confounding effects on estimations of effective fecunditity relative to spatial sampling biases and to the spatial heterogeneity of plant distribution (potentially impacting competition among males), while our spaMM procedure controls for spatial environmental heterogeneity.

We applied our methodology to Leucadendron rubrum, a dioecious wind-pollinated serotinous shrub, endemic to the fire-prone South-African fynbos. L. rubrum displays extreme sexual dimorphism (Fig. S1), with males being typically more highly branched, having smaller leaves and taller stature than females (Harris \& Pannell, 2010; Welsford et al., 2014; Welsford et al., 2016). A single recruitment pulse typically occurs after fire, killing all adult trees and releasing seeds stored in their canopy seed bank (Cowling \& Lamont, 1987). This particular life-cycle allows 
171 the estimation of life-time effective fecundity by sampling seedlings only once (i.e. after the fire 172 event). Furthermore, because recruitment is synchronized by fire, all sampled adult individuals in 173 the population have the same age (Bond, 1984). The strong sexual dimorphism of L. rubrum has 174 been previously hypothesized to be the consequence of sex-specific resource requirements (Harris \& 175 Pannell, 2010). Indeed, the cost of reproduction in L. rubrum is likely to differ strongly between 176 sexes due to the cost of maintaining the canopy-stored ('serotinous') seed bank in females (Martín177 Sanz et al., 2017). We hypothesized that, because of such maternal care, female fitness may be more 178 sensitive to water availability than male fitness. Owing to these differences in resource requirement 179 for reproduction, we therefore tested whether male and female effective fecundities (as defined 180 above) display different spatial structure and whether the observed strong sexual dimorphism is 181 associated with sex-specific selection gradients (Lande, 1980). 


\section{Materials and methods}

183

\section{Study species and site}

Leucadendron rubrum is a dioecious wind-pollinated shrub species endemic to the Western Cape of South Africa (Rebelo, 2001) where natural fires occur every 10-15 years (van Wilgen et al., 2010; Kraaij et al., 2011). L. rubrum belongs to the Proteaceae family and flowers from August to September. Seed recruitment is constrained to a short period following fires, and seeds released between fires typically fail to establish due to competition (Bond, 1984). L. rubrum typically starts flowering at 2-3 years, and seeds are retained in woody cones for several years (Harris \& Pannell, 2010). Seeds therefore form a 'serotinous' seed bank, which persists until fire kills the plants, allowing cone opening and wind dispersal of fruits via their plumed perianth (Williams, 1972; Rebelo, 2001). In serotinous species, disruption of water intake to the cone (caused by broken branch or plant death) was shown to lead to seed release suggesting a water cost to keep the cones closed (Treurnitcht et al., 2016; Martín-Sanz et al., 2017). Thus, mother death or any event leading to cone opening before the advent of fire results in seed release in poor conditions for recruitment and ultimately in the loss of progeny.

The study population was located at Bainskloof pass (33³2'21.25"S 19¹0'12.10"E) and was contained in a rectangle of 135x110 meters (Fig. S2). We studied all adult individuals of our focal population. Another population of $L$. rubrum was located at a distance of 310 meters (smallest distance found between two plants from the two populations). All adults of L. rubrum of the focal population (i.e. 86 females and 88 males) were mapped (see Supplementary Methods S1), sampled for DNA analyses and measured for several traits in 2004. In summer 2006, a fire burnt the population, killing all adults. A total of 1,265 seedlings were mapped, and their leaves were sampled in the following Fall (February 2007), four to five months after the fire. The spatial distribution of adults and seedlings is shown in Fig. S2. In one part of the study site, a ditch had been dug for construction after seeds had dispersed, so we were unable to determine the undisturbed positions of 
172 seedlings located in that area which we therefore eliminated from the dataset. However, the

effective fecundity of adults in this area can still be estimated without bias induced by the disturbance, thanks to the use of MEMMseedlings (see below).

When sampling seedlings, the presence of seedlings from another closely related sympatric species (Leucadendron salignum P.J. Bergins) rendered the identification of L. rubrum juveniles difficult. To ensure that only juveniles of L. rubrum were included in later analyses, we genotyped juveniles (see below for details on the genotyping protocol), as well as adults from both species. This analysis aimed at assigning seedlings to either species and its results are described in Supplementary Method S2 and Fig. S3. We did not find evidence for the existence of hybrids between $L$. rubrum and L. salignum. In addition, 254 juveniles were excluded after genotyping as they belonged to L. salignum.

\section{Measurements of adult traits in the field}

For adult shrubs, we measured in 2004 three traits describing plant architecture and three traits describing leaf morphology (available at doi:10.5061/dryad.ngf1vhhst). All six traits are known to be sexually dimorphic in this species (Harris \& Pannell, 2010; Welsford et al., 2014, 2016; Fig. S4). The three traits describing plant architecture were (1) plant height, (2) the first diameter defined as the greatest horizontal diameter of the plant (hereafter, canopy diameter), and (3) the second horizontal diameter defined as the diameter perpendicular to the first diameter (hereafter, second diameter). Several leaves were collected randomly along branches of each adult, dried and photographed. Pictures were analyzed to measure the three traits describing leaf morphology: (1) leaf area, (2) length and (3) width using ImageJ (Schneider et al., 2012). The number of leaves analyzed per adult ranged from 10 to 23 with an average of 20.3. In females, we counted the number of cones in the last two cohorts (cones produced in the last two seasons of cone production and maintained closed since). Because older cohorts were not counted, this measure reflects cone 
233 production rather than the maintenance of the serotinous seed bank. We did not count male cones 234 because they were too numerous.

235

\section{Microsatellite genotyping}

We genotyped both adults and their progeny in our focal population (available at doi:10.5061/dryad.ngf1vhhst). For both adults and seedlings, sampled leaves were preserved in silica gel prior to DNA extraction using a modified version of the CTAB protocol (Justy et al., 2009). We designed two polymerase chain reaction (PCR) multiplexes for amplifying DNA at 4 microsatellite loci, each involving primers with different fluorescent labels (Multiplex 1: 4F8, 3C9, 1C7, 1C3; Multiplex 2: 3B11, 2B2, 1D7, 1B8; markers developed by Justy et al., 2009). PCRs were performed using the Qiagen Multiplex PCR Kit (Qiagen, Hilden, Germany); each PCR reaction was performed in a final volume of $10 \mu \mathrm{M}$ composed of $5 \mu \mathrm{L}$ of Multiplex Master mix $(2 \mathrm{X}), 1 \mu \mathrm{L}$ of primer sequences $(1 \mu \mathrm{M}), 1 \mu \mathrm{L}$ of DNA extracts and $3 \mu \mathrm{L}$ of sterile water. PCR reactions were performed on a Mastercycler pro thermocycler (Eppendorf, vapo.protect) with an initial denaturation step of $15 \mathrm{~min}$ at $95^{\circ} \mathrm{C}, 35$ cycles of 30 s at $94{ }^{\circ} \mathrm{C}, 1 \mathrm{~min} 30$ s at the $\mathrm{T}_{\mathrm{m}}$ temperature (M1: $54^{\circ} \mathrm{C}, \mathrm{M} 2: 53^{\circ} \mathrm{C}$ ) and $1 \mathrm{~min}$ at $72{ }^{\circ} \mathrm{C}$, and a final step of $30 \mathrm{~min}$ at $60^{\circ} \mathrm{C}$. Genotyping was performed on an ABI3500XL sequencer. The genotypes of all adults and offspring were scored using GeneMapper at the eight microsatellite loci, which exhibited between five and 23 alleles (Table S1 for information per marker). After excluding individuals that did not amplify, our dataset contained 82 females, 85 males and 869 juveniles, corresponding to an amplification failure of roughly $8 \%$ for both adults and juveniles. For each microsatellite marker, we used CERVUS (version 3.0.7; Kalinowski et al., 2007) to estimate the non-exclusion probabilities of the first parent, the second parent and parent pairs, to test for Hardy-Weinberg equilibrium, and to compute null allele frequencies (Table S1). Non-exclusion probabilities correspond to the probabilities that the set of loci will not exclude an unrelated candidate parent (or parent pair) of an arbitrary offspring. Finally,

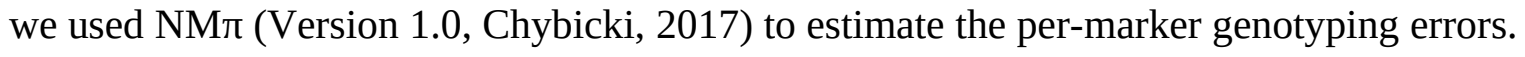




\section{Joint estimation of effective fecundities and both pollen and seed dispersal kernels}

We used a method that uses information about the genotype and the spatial location of adults and seedlings to jointly estimate pollen and seed dispersal kernels and the individual male and female effective fecundities - a proxy for fitness (see the Introduction). Our method builds on the Mixed Effects Mating Model, MEMMseedlings (Oddou-Muratorio et al., 2018), which models mating and dispersal events in a hermaphroditic plant population to estimate the selfing rate, immigration rates and dispersal kernels for both pollen and seeds as well as the variance in male and female effective fecundity (i.e. using random individual effects). We modified the MEMMseedlings algorithm to produce a new version that handles separate sexes, with distinct spatial distributions of male and female plants (see Supplementary Method S3, available at https://gitlab.paca.inrae.fr/pub/tonnabel_mol_ecol.).

MEMMseedlings takes into account both the variation in fecundity among individuals and the relative positions of putative parents and seedlings when computing the likelihood of observed genotypes conditional to dispersal parameter and individual fecundity estimations. In MEMMseedlings, putative parents that are more distant from a seedling have a lower parentage probability. This model also describes mate competition through a mass action law, i.e. the contribution of a given male to the pollen cloud of a given female is diluted among the contributions of all other males. For these reasons, the model can estimate variation in effective fecundities, separately from different sources of spatial variation in reproductive success, linked to (i) spatial biases in seedling sampling, (ii) the spatial distribution of mates, or (iii) edge effects. Accordingly, we checked that the estimated effective fecundity of adult plants located on the border of our study population was not different than elsewhere in the study population by generalized linear mixed models (results not shown). Our model assumes the same pollen (or seed) dispersal kernels for each male (or respectively female) individual, and isotropic wind dispersal patterns. Given that anisotropy can sometimes be found in wind-pollinated plants (Austerlitz et al. 2007), we confirmed 
an absence of signal for anisotropy using NMr (see Supplementary Method S3). MEMMseedlings

estimates a relative measure of effective fecundity, scaled by the average effective fecundity, (see equation (1) in Supplementary methods S3), which is therefore unitless.

Given the life cycle of $L$. rubrum and the sampling of seedlings after the fire, our estimated effective fecundity integrates the effect of variation between individuals, either male or female, not only for pollen and ovule production, but also for pollen export, fertilization rate, seed maturation and dispersal, maintenance of seeds within the cones (degree of serotiny), adult survival until the fire, seed germination and juvenile survival until the seedling census. Seeds released after the fire and previously stored in the canopy were potentially fertilized in different years. Because adults of L. rubrum do not survive fire, the establishment of progeny after a fire thus represents their entire lifetime reproduction.

Briefly, our MEMMseedlings model combines genotypes data and spatial distribution data for both adults and offspring to estimate, in a Bayesian framework, individual male and female effective fecundities ( $F_{k}$ and $R_{j}$, respectively), the seed immigration rate $\left(m_{s}\right)$, the pollen immigration rate $\left(m_{p}\right)$, the rate of pollen export to the pollen cloud of non-local mothers $(v)$, the mean seed dispersal distance $\left(\delta_{s}\right)$, the mean pollen dispersal distance $\left(\delta_{p}\right)$, a parameter affecting the shape of the seed dispersal kernel $\left(b_{s}\right)$ and that of the pollen dispersal kernel $\left(b_{p}\right)$. The estimation of pollen and seed immigration rates $\left(m_{s}\right.$ and $\left.m_{p}\right)$ and, the rate of pollen export to the pollen cloud of non-local mothers, $(v)$ depends on the actual process of immigration, but is also affected by the fact that around $8 \%$ of the parents were excluded from the analysis due to a lack of amplification. Immigration rates therefore include the probabilities of maternity and paternity attributable to unsampled parents. Both pollen and seed immigration rates are therefore likely overestimated. Finally, when computing Mendelian transition probabilities between seedlings and putative parents, MEMMseedlings considers genotyping errors by allowing a parent-offspring genetic discrepancy at a maximum of one locus, and at each locus, the probability to mistype any allele was fixed using the per-marker genotyping error rates estimated by NMт (see Supplementary Method S3). To describe 
311 the quality of parentage assignments associated with the dataset, MEMMseedlings computes the posterior probabilities for all seedlings for which (i) both parents are known among the genotyped 
337 for different spatial distributions of random effects between sexes (publicly available in spaMM 338 since version 2.6.0). The classical Matérn correlation function was used to model the spatial autocorrelation of random effects, separately for each sex, as a function of the distance between individuals. The Matérn correlation model involves two parameters: the smoothness parameter $(v)$, and the scaling parameter for distances $(\rho)$. We compared models fits considering (1) no spatial autocorrelation, (2) the same spatial autocorrelation for both sexes and (3) two sex-specific spatial autocorrelation structures. We used likelihood ratio tests (LRTs) to (i) first test for a sex-specific spatial structure in morphology, by comparing models with a different structure of random effects but the same fixed effects, and (ii) to test for sexual dimorphism, by comparing the selected model in step (i) to a model fitted with a similar structure of random effects, but without sex as a fixed effect. Models were fitted either by maximum likelihood, for performing LRTs between models differing in their fixed-effects structure, or by restricted maximum likelihood, for LRTs between models differing in their random-effect structures and for computing the predictions from the model fits. We also examined whether male and female plants were spatially segregated (i.e. whether the sex ratio was spatially autocorrelated) using a binomial generalized linear mixed model (GLMM) with sex as the response variable and compared model fits with or without a spatially autocorrelated random effect as described above. Finally, we tested whether the number of cones produced by females displayed a non-uniform spatial distribution by comparing models including cone number as the response variable, plant density (see below for its calculation) as a fixed factor, and either a spatial random effect or not.

\section{Multivariate sex-specific selection analysis}

Inspired by the multivariate framework of Lande and Arnold (1983), we examined in a single full model the relationship between the relative effective fecundity as the response variable and, the following explanatory variables: canopy diameter, leaf area, plant density, sex, and the interaction between each of the three former explanatory variables and sex. We fitted generalized linear mixed- 
effects models (Gamma GLMM with logarithm link function) to describe the variation in effective fecundity, while including spatially autocorrelated random effects. We checked that a model predicting effective fecundity using a Gamma distribution with a log link performed better than models assuming a different candidate distribution (compare gamma log link: cAIC=18.7 with gaussian with a link identity: $\mathrm{cAIC}=139$; or gaussian with a log link: cAIC $=126$ - cAIC is a metric similar to the traditional $A I C$, except that it measures prediction performance conditionally on the realization of the random effects, Vaida \& Blanchard, 2005). We followed the same procedure as described for the analysis of sexual dimorphism (see previous section) to fit the models using the $\mathrm{R}$ package spaMM and to identify the best fitting random spatial structure (i.e. comparing a sexspecific spatial structure, a common spatial structure and a lack of it). We were thus able to compare the patterns of spatial variation of residual effective fecundity between males and females, once the effects of local density and variation in morphology were included as fixed effects in our global model. Spatial autocorrelation in effective fecundity may then reflect spatial variation in unmeasured environmental variables (such as water availability) or unmeasured plant traits. Different spatial structures between sexes therefore inform on the differential sensitivities of their effective fecundities to variation in such unmeasured variables. We took into account the variation in uncertainty of the individual effective fecundity estimates by parameterizing the residual variance as a function of the variance in MCMC estimates of individual fecundity during the fitting (see Supplementary Method S4). We again fitted models with distinct residual variances for each sex. Because our MEMMseedlings model estimated large effective fecundity for several plants located in one part of the study population, we performed a sensitivity analysis testing the robustness of our results to the removal of all individuals with standardized effective fecundity greater than four standard errors (for all statistical models treating effective fecundity as response variable). Five females and one male were removed in this sensitivity analysis, including four individuals that were particularly close from each other. 
We chose to investigate the selection gradients for two morphological predictors only

(canopy diameter and leaf area), because several of the six morphological traits were highly correlated (Table S3). Notably, all three traits describing plant architecture were strongly correlated with each other (i.e. plant height, canopy diameter and second diameter, Table S3). Similarly, our three measures of leaf morphology were strongly correlated with each other (i.e. leaf area, length and width, Table S3). Estimation of multivariate selection gradients and their interpretation could be confused by such strong correlations between traits (see Chong et al., 2018 for a review). We therefore retained only two morphological traits in our selection analysis (but similar results were obtained for other combinations of traits). We standardized the two focal traits using z-scores based on the mean and variance values of the traits calculated separately for each sex. We confirmed that leaf area significantly differed between individuals by comparing, within each sex, models predicting leaf area with vs. without an individual-level random effect using an exact restricted likelihood ratio test (Crainiceanu and Ruppert, 2004) implemented in package RLRsim 3.1-6 (Scheipl et al., 2008): for females, likelihood ratio (LR)=1573.9, $\mathrm{p}<0.001$; for males, LR=625.1, $\mathrm{p}<0.001$.

We compared the performance of local plant density measured at different scales in explaining variation in male and female effective fecundity (see Supplementary Method S5). We did not transform plant density into z-scores since this predictor is not an individual trait and thus its associated slope should not be considered as a selection gradient. To compare models explaining variation in effective fecundity with different scales used to compute plant density, we used cAIC. The best model included the density of plants in a quadrat of $12 \times 12 \mathrm{~m}$ around the focal individual. We therefore retained this metric to compute the values of plant density used in our all subsequent analyses.

To test for the significance of selection gradients, we first compared the fit of the full model to the fit of nested models in which one of the three predictors of interest (i.e. canopy diameter, leaf area and plant density) had been removed, both as a main effect and in interactions. This revealed 
414 whether there was any effect of the focal predictor. Second, we compared the full model to models 415 in which only the interaction between sex and one of the three variables of interest had been 416 removed. This allowed us to test whether the effect of the predictor was different between sexes. We 417 also built separate GLMMs for each sex, predicting effective fecundity from the three predictors of 418 interest. We used these sex-specific models to test the effect of each predictor in each sex, if and 419 only if, the interaction between sex and a given predictor was significant. We compared the fit of 420 our full model, which considers linear selection gradients only, to the fit of a similar model 421 including quadratic and correlational selection terms for canopy diameter and leaf area, and to 422 model fits including one quadratic or correlational term at a time.

We also estimated selection gradients for the same morphological traits (canopy diameter 424 and leaf area), using as a proxy for fitness, not the estimated effective fecundity but the actual 425 number of cones empirically counted on female plants. We predicted the number of female cones 426 from traits using a Poisson GLMM with spatially autocorrelated random effects and plant density as 427 a covariate in addition to the two focal traits. Finally, we predicted the female effective fecundity 428 from the number of empirically counted cones, also with spatially autocorrelated random effects and 429 plant density as a covariate. 


\section{Results}

\section{L. rubrum showed strong sexual dimorphism in morphology}


distance-dependent pollen dispersal nevertheless explained our data better than modeling a uniform distribution of pollen dispersal distances (Fig. S7, see Supplementary Methods S3 for a description of their comparison). Although we estimated a high probability for the pollen to travel long distance, we estimated that about three quarters of the seedlings had a genotyped father in the population (1$m_{p}=0.85$ for seedlings with a known mother or $v=0.81$ for seedlings with an unknown mother), which is similar to the proportion found for mothers $\left(1-m_{s}=0.89\right.$; Table 1$)$. Estimations of dispersal kernels with the $\mathrm{NM \pi}$ algorithm yielded similar parameter estimation, yet with a notable shorter estimate of mean pollen dispersal distance (Table 1 vs. S2). The estimates of dispersal kernels with $\mathrm{NM \pi}$ were robust to the inclusion of anisotropy in dispersal events (results not shown).

\section{Autocorrelation in effective fecundity occurred on a smaller spatial scale for males}

\section{than females}

Effective fecundity estimations were carried out using a set of 8 microsatellite markers containing between 6 and 24 alleles, showing non-exclusion probabilities of parent pairs ranging between 0.12 to 0.58 and genotyping error rates ranging from $0.9 \%$ to $5.5 \%$ (Table S1). The combination of the genotype data and plant spatial distribution data provided information to assign two parents to 88\% of seedlings (Figure SM3 in Supplementary Methods S3).

We detected a clear sex-specific spatial structure for effective fecundity (Table S5). We found different spatial variation in effective fecundity for each sex, with coarse-grained and finegrained spatial effects for females and males, respectively (Fig. 3a vs. 3b). These results were robust to the removal of plants with standardized effective fecundity greater than four standard errors, which only slightly affected the previous conclusion (Table S5; Fig. S8). Several plants with large effective fecundity were found in the disturbed area (Fig. 3). The number of empirically counted cones on female plants also displayed a significant spatial autocorrelation $\left(\chi^{2}=2238, d f=3, p<0.0001\right.$; Fig. S9). Cone number was significantly correlated with relative effective fecundity but the effect size was small (using standardized cone number, $\beta=0.286, \chi^{2}=6.02, d f=1, p=0.0141$ ) and the two spatial distributions were not fully aligned (Fig. 3a vs. S9). 
Selection for higher leaf area and wider canopies similar in both sexes

485

486

487

488

489

490

491

492

493

494

495

496

497

498

499

500

501

502

503

504

Our spatially corrected selection gradient approach revealed that the leaf area was negatively associated with effective fecundity (Table 2 and S5; Fig. 4), with similar slopes in both sexes as shown by a non-significant interaction between leaf area and sex (Table S5). Yet, the leaf area was positively correlated to the number of counted cones on female plants $\left(\beta=0.171\right.$; LRT: $\chi^{2}=42.7$, $d f=1, p<0.001)$. Larger canopy diameter was significantly associated with higher effective fecundity (Table 2 and S5; Fig. 4). Accordingly, female plants with wider canopies displayed higher numbers of empirically counted cones $\left(\beta=0.904\right.$; LRT: $\left.\chi^{2}=78.8, d f=1, p<0.001\right)$. The increase in effective fecundity with increasing canopy diameter was similar in both sexes, as shown by a non-significant interaction between sex and canopy diameter (Table S5). Our estimates of selection gradients were robust to the removal of plants with effective fecundity greater than four standard errors (Table S5; Fig. S10). We found no signal for non-linear selection, either by including all three quadratic and correlational terms at once (LRTs: $\chi^{2}=0.709, \mathrm{df}=3, p=0.871$ ), or each of them separately ((leaf area $)^{2}: \chi^{2}=0.129, d f=1, p=0.719$; $(\text { canopy diameter })^{2}: \chi^{2}=0.118, \mathrm{df}=1, \mathrm{p}=0.731$; leaf area x canopy diameter: $\left.\chi^{2}=0.378, \mathrm{df}=1, \mathrm{p}=0.539\right)$.

The effect of plant density on effective fecundity (Fig. S6) differed between sexes, as revealed by a significant interaction between sex and plant density (Table S5; Fig. 4c). Plant density was negatively associated with male effective fecundity, but female effective fecundity showed no association with plant density (Table S5). The effect of the interaction between sex and plant density was however only marginally significant when the plants with the highest effective fecundity were removed in the robustness analysis (Table S5; Fig. S10). 


\section{Novel methods for dealing with spatial bias affecting selection estimates in plants}

510

Technical and methodological improvements in parentage assignations now allow for estimation of plant fitness in natural populations from genetic data, and provide the link between fitness and plant traits through selection gradients analyses (e.g. Meagher \& Thompson, 1986; Burczyk \& Prat, 1997; Burczyk et al., 2006; van Kleunen \& Burczyk, 2008). We have developed a methodology that estimates effective fecundity in dioecious plants while accounting for biases associated with their spatial distribution. Beyond the interest of documenting spatial patterns of seed and pollen dispersal, the addition of this spatially explicit component to classical parentage methods (Jones, 2010) improves the estimation of effective fecundity in the presence of confounding effects, such as spatial bias in sampling descendants, spatial variation in the intensity of mate competition triggered by a non-uniform distribution of mates, or border effects (Oddou-Muratorio et al., 2018). We investigated effects of traits on effective fecundity by classical selection gradient methods (Lande \& Arnold, 1983), in which we explicitly modeled the effect of spatially correlated unmeasured environmental factors on effective fecundity. This newly developed framework will be particularly suited to estimating selection in natural populations, given that spatial biases are typically difficult to avoid regarding both sampling and uncontrolled factors.

\section{No contemporary sexually antagonistic selection despite strong sexual dimorphism}

The signal we found for selection of larger canopy diameters in both sexes may indicate a 'budget effect' of plant size, where larger plants acquire more resources that can be reallocated to gamete production (Delph \& Ashman, 2006). In females, the number of counted cones was positively related to canopy diameter. However, cone number only poorly explained effective fecundity. The spatial distribution of cone number and female effective fecundity were moreover not fully matching, suggesting that processes occurring after cone production, such as cone maintenance or mother plant survival affect the female effective fecundity. In males, selection for wider canopies 
could be linked to flower production if both sexes are subject to similar ontogenetic constraints. We

534 found evidence for similar selection for smaller leaf area in both sexes. Smaller leaves were

previously shown to be correlated in L. rubrum with thinner, and more numerous, branches and less efficient water transport from roots to branch apex (Harris, 2007). Selection for smaller leaves in males may reflect selection for greater number of inflorescences held on more flexible branches, a trait long hypothesized to enhance pollen dispersal (Klinkhamer et al., 1997). Smaller leaves may also represent a decreased mechanical hindrance to pollen dispersal. Selection for smaller leaves in females, however, contradicts our expectation of selection for enhanced water transport to the cones. The number of cones counted on females was furthermore positively related to leaf area. The fact that leaf area relates to the number of cones, and to effective fecundity in opposite ways, suggests that any positive effects of leaf area on fecundity through female cone production may have been masked by trade-offs with other key life history components for serotinous plants (e.g. adult survival until the fire). In the absence of positive genetic correlation between sexes, sexual conflicts may be resolved once each sex reaches its respective optimum. We however did not find evidence for stabilizing selection in the study population, which one could expect if each sex was at its optimum with sufficient genetic variation in the studied traits.

Only $17 \%$ of studies estimating selection gradients in animals identified sexually antagonistic selection (Cox \& Calsbeek, 2009). The paucity of evidence for sexually antagonistic selection, with which our study concurs, is inconsistent with the idea that genetic correlations between sexes should maintain sexually antagonistic selection over long periods of time (Lande, 1980). To explain this inconsistency, both theory and experiments have suggested that temporal or spatial ecological changes may result in variable pattern of selection acting on males and females, with both sexes displaying trait values remaining far away from their ever changing ecological optimum (Kokko \& Rankin, 2006; Delph et al., 2011; Sheridan \& Bickford, 2011; Long et al., 2012; Berger et al., 2014; Connallon, 2015; Connallon \& Hall, 2016). Recent theoretical 
developments showed that positive inter-sexual covariance for resource acquisition traits could also impede the identification of sexually antagonistic selection (Zafitschek \& Connallon, 2017).

An alternative explanation to the lack of detected antagonistic selection is that antagonistic selection is present but masked by a positive correlation between a locally varying unmeasured ecological factor and both effective fecundity and a focal morphological trait (Price et al., 1988). A large body of theoretical work also suggests that the combination of a strong sexual dimorphism with a lack of sexually antagonistic selection found in L. rubrum may result from adaptation to changing ecological conditions, which cause patterns of selection between sexes to align. A longer fire return interval, or low resource availability, are predicted to select for increased resource allocation to plant survival and a lower allocation to cone maintenance (Tonnabel et al., 2012). That is because serotinous plants need to survive until fire to reproduce and can therefore afford to maintain cones over long periods of time only if it does not come at the expense of their own survival up until the fire. These particular ecological conditions may have weakened selection in females for traits improving current water conductance and cone maintenance, and favored instead traits improving cone production and adult survival. Understanding the emergence of sexual dimorphism through sex-specific selection estimations will therefore require either experimental protocols controlling environmental conditions or extensive estimations of spatio-temporal variation in sex-specific selection in natural populations. Long-term studies in animals have indeed commonly found large temporal variation in selection pressures acting on various traits (e.g. Grant \& Grant, 2002; Reimchen \& Nosil, 2002; Reed et al., 2013; Acker et al., 2015), including both temporal and spatial variation in the direction of sexually antagonist selection pressure on sexually dimorphic traits (Fargevieille, 2016).

\section{Sex-specific spatial distribution of effective fecundities}

The observation that reproductive costs differ between males and females (e.g. Antos \& Allen, 1990; McDowell et al., 2000; Harris \& Pannell, 2008; van Drunen \& Dorken, 2012) has been pivotal to discussions on the evolution of sexual dimorphism in plants (Freeman et al., 1976; Delph 
\& Bell, 2008). Resources key to male and female reproduction commonly display small-scale

variation in the wild (Silvertown et al., 1999; Araya et al., 2011). Therefore, it is a simple corollary of the sex-specific cost of reproduction hypothesis that male and female fitness should often exhibit different spatial patterns in natural populations, as found in our study population. These sex-specific spatial patterns of fitness variation call for future studies relating small-scale variation in key resource types (Silvertown et al., 1999; Araya et al., 2011) and plant fitness in both males and females.

\section{Only male effective fecundity was affected by density}

Male effective fecundity was negatively associated with plant density while no association was found for females. The negative effect of plant density on male effective fecundity might be triggered by increased competition over nutritive resources affecting pollen production, by negative effects of a closed canopy on pollen dispersal, or by competition effects at the seedling stage affecting their offspring. The lack of an effect of plant density on female reproduction suggests that, either they are less affected than males by competition with other plants, or that the negative effects of competition are counter-balanced by positive effects of reproducing in a high density patch. In both cases, it suggests different reproductive needs and ecology in males and females. Plant density was shown to negatively affect both male and female effective fecundity in a wind-pollinated tree (Oddou-Muratorio et al., 2018), and was also shown to impede pollen dispersal in a wind-pollinated herb (Tonnabel et al., 2019b). We note however that this sex-specific effect of density on effective density was only marginally significant when removing individuals with large fecundity.

\section{Pollen and seed dispersal kernels typical of plant dispersal behaviour}

Our spatially explicit method allowed the estimation of dispersal kernels, which revealed a fat-tailed seed dispersal kernel in the anemochorous L. rubrum. Most seeds dispersed close to the mother plant, but some fraction dispersed much further. Similarly, a meta-analysis including species from various plant families, continents, vegetation types and growth forms found a predominance of fattailed seed dispersal kernels (Bullock et al., 2017). Investigations of pollen dispersal kernels are 
610 scarce, but they typically also indicate fat-tailed kernels in both insect- (Austerlitz et al., 2004;

611 Oddou-Muratorio et al., 2005; but see Matter et al., 2013) and wind-pollinated species (Austerlitz et al., 2004; Goto et al., 2006; Gaüzere et al., 2013; Geber et al., 2014 but see Ahee et al., 2015). Our estimated pollen dispersal kernel showed a markedly fat-tailed distribution, whereby a large proportion of pollen was able to disperse over large distances; similar large distance pollen dispersal was reported in both wind-pollinated and animal-pollinated species (e.g. Devaux et al., 2005; O’Connell et al., 2007). Given the large estimates of pollen dispersal distances and the short distance to the nearest population, the low estimates of pollen immigration are unexpected, especially given the same order of magnitude as seed immigration rate. This discrepancy is nevertheless consistent with other studies showing that the amount of long distance dispersal inferred by spatially explicit parentage modeling is not always congruent with the amount predicted by dispersal kernels inferred from local dispersal events (Chybicki \& Oleksa, 2018; Hardy et al., 2019). Such inconsistencies may emerge when extrapolation of dispersal kernels does not properly account for an increased probability of encountering obstacles between populations.

In conclusion, we found sex-specific variation in fitness in a natural population of a highly dimorphic plant species, despite similar directional selection in both sexes. Plant density impacted males and females differently, suggesting that sexes may display different sensitivities to competition over resources, regardless of competition for mates. The fixed life-form of plants might often generate spatial structure in fitness, as displayed in our study population. We therefore advocate for the generalization of spatial methods for estimating selection gradients, combined with spatially explicit fitness estimation methods for estimating selection acting on plants in the wild. In the long run, such methods should also account for the sex-specific temporal variation in plant phenology and the relatedness between potential mates as both can also influence fitness (Ismail \& Kokko, 2019) and are likely to show spatial patterns. 


\section{Acknowledgments}

635

636

637

analysis, to John Pannell for helpful discussions and English editing, to Jeremy Midgley for help in

640 the field and attracting our interest in this system. Elodie Flaven-Noguier, Fabienne Justy and

641 Clémence Hatt helped with the DNA extractions, genotyping and molecular biology protocols. This

642 work was supported by a grant from the FRB to IO and AM, from the ANR “Evorange”, ANR-09PEXT-011 to OR and from the ANR "MeCC”, ANR-13-ADAP-006 to OR, EKK and SOM. JT was 644 supported by a grant to John Pannell from the FNS, 31003A_163384. OR acknowledges support 645 from the Peter Wall Institute of Advanced Studies, UBC, and from CNRS. 


\section{References}

Acker P, Grégoire A, Rat M, Spottiswoode CN, van Dijk RE, Paquet M, Kaden JC, Pradel R, Hatchwell BJ, Covas R, Doutrelant C. 2015. Disruptive viability selection on a black plumage trait associated with dominance. Journal of Evolutionary Biology 28: 2027-2041.

Ahee JE, Van Drunen WE, Dorken ME. 2015. Analysis of pollination neighbourhood size using spatial analysis of pollen and seed production in broadleaf cattail (Typha latifolia). Botany 93: 91-100.

Antos JA, Allen GA. 1990. A comparison of reproductive effort in the dioecious shrub Oemleria cerasiformis using nitrogen, energy and biomass as currencies. The American Midland Naturalist 124: 254-262.

Araya YN, Silvertown J, Gowing DJ, McConway KJ, Linder HP, Midgley G. 2011. A fundamental, eco-hydrological basis for niche segregation in plant communities. New Phytologist 189: 253-258.

Arnold SJ. 1994. Bateman’s principles and the measurement of sexual selection in plants and animals. The American Naturalist 144: S126-S149.

Austerlitz F, Dick CW, Dutech C, Klein EK, Oddou-Muratorio S, Smouse PE, Sork VL. 2004. Using genetic markers to estimate the pollen dispersal curve. Molecular Ecology 13: 937954.

Austerlitz F, Dutech C, Smouse PE, Davis F, Sork VL. 2007. Estimating anisotropic pollen dispersal: a case study in Quercus lobata. Heredity 99: 193-204.

Barrio M, Teixido AL. (2015) Sex-dependent selection on flower size in a large-flowered Mediterranen specie: experimental approach with Cistus ladanifer. Plant Systematics and Evolution 301:113-124.

Bateman AJ. 1948. Intra-sexual selection in Drosophila. Heredity 2: 349-368. 
695 Connallon T. 2015. The geography of sex-specific selection, local adaptation, and sexual 696 
Dorken ME, Perry LE. 2017. Correlated paternity measures mate monopolization and scales with 
Goto S, Shimatani K, Yoshimaru H, Takahashi Y. 2006. Fat-tailed gene flow in the dioecious canopy tree species fraxinus mandshurica var. japonica revealed by microsatellites. Molecular Ecology 15: 2985-2996. 
Justy F, Midgley J, Olivieri I. 2009. Isolation and characterization of ten microsatellites markers in Leucadendron rubrum (Proteaceae). Molecular Ecology Resources database 9: 1375-1429.

Kalinowski ST, Taper ML, Marshall TC. 2007. Revising how the computer program CERVUS accommodates genotyping error increases success in paternity assignment. Molecular Ecology 16: 1099-1106.

Klein EK, Desassis N, Oddou-Muratorio S. 2008. Pollen flow in the wildservice tree, Sorbus torminalis (L.) Crantz. IV. Whole interindividual variance of male fecundity estimated jointly with the dispersal kernel. Molecular Ecology 17: 3323-3336.

Klein EK, Bontemps A, Oddou-Muratorio S. 2013. Seed dispersal kernels estimated from genotypes of established seedlings: does density dependent mortality matter? Methods in ecology and Evolution 4:1059-1069.

Klinkhamer PGL, de Jong TJ, Metz H. 1997. Sex and size in cosexual plants. Trends in Ecology and Evolution 12: 260-265.

Kokko H, Rankin DJ. 2006. Lonely hearts or sex in the city? Density- dependent effects in mating systems. Philosophical Transaction of the Royal Society B 361: 319-334.

Kraaij T, Cowling RM, van Wilgen BW. 2011. Past approaches and future challenges to the management of fire and invasive alien plants in the new Garden Route National Park. South African Journal of Science 107: doi:10.4102/sajs.v107i9/10.633.

Lande R. 1980. Sexual dimorphism, sexual selection and adaptation in polygenic characters. Evolution 34: 292-305.

Lande R, Arnold SJ. 1983. The measurement of selection on correlated characters. Evolution 37: $1210-1226$. 
Oddou-Muratorio S, Gauzère J, Bontemps A, Rey J-F, Klein EK. 2018. Tree, sex and size: ecological determinants of male vs. female fecundity in three Fagus sylvatica stands. Molecular Ecology 27:3131-3145. 
Renner SS. 2014. The relative and absolute frequencies of angiosperm sexual systems: Dioecy, monoecy, gynodioecy, and an updated online database. American Journal of Botany 101: 1588-1596.

Rousset F, Ferdy J-B. 2014. Testing environmental and genetic effects in the presence of spatial autocorrelation. Ecography 37: 781-790.

Scheipl F, Greven S, Kuechenhoff H. 2008. Size and power of tests for a zero random effect variance or polynomial regression in additive and linear mixed models. Computational Statistics \& Data Analysis 52:3283-3299.

Schiestl FP, Johnson SD. 2013. Pollinator mediated evolution of floral signals. Trends in Ecology and Evolution 28: 307-315.

Schneider CA, Rasband WS, Eliceiri KW. 2012. NIH Image to ImageJ: 25 years of image analysis. Nature methods 9: 671-675.

Schoen DJ, Stewart SC. 1987. Variation in male fertilities and pairwise mating probabilities in Picea glauca. Genetics 116: 141-152.

Sheridan JA, Bickford D. 2011. Shrinking body size as an ecological response to climate change. Nature Climate Change 1: 401-406.

Silvertown J, Dood ME, Gowing DJG, Mountford JO. 1999. Hydrologically defined niches reveal a basis for species richness in plant communities. Nature 400: 61-63.

Tonnabel J, Van Dooren T, Midgley J, Haccou P, Mignot A, Ronce O, Olivieri I. 2012. Optimal resource allocation in a serotinous non-sprouting plant species under different fire regimes. Journal of Ecology 100: 1464-1474. 
Tonnabel J, Mignot A, Douzery EJP, Rebelo T, Schurr F, Midgley J, Illing N, Justy F, Orcel D, Olivieri I. 2014. Convergent and correlated evolution of major life-history traits in the angiosperm genus Leucadendron (Proteaceae). Evolution 68: 2775-2792.

Tonnabel J, David P, Pannell JR. 2017. Sex-specific strategies of resource allocation in response to competition for light in a dioecious plant. Oecologia 185:675-686.

Tonnabel J, David P, Pannell JR. 2019a. Do metrics of sexual selection conform to Bateman's principles in a wind-pollinated plant? Proceedings of the Royal Society, B 20190532.

Tonnabel J, David P, Klein EK, Pannell JR. 2019b. Sex-specific selection on plant architecture through 'budget' and 'direct' effects in experimental populations of a wind pollinated herb. Evolution 73:897-912.

Treurnicht M, Pagel J, Esler KJ, Schutte-Vlok A-L, Nottebrock H, Rebelo AG, Schurr FM. 2016. Environmental drivers of demographic variation across the global geographical range of 26 plant species. Journal of Ecology 104: 331-342.

Vaida F, Blanchard S. 2005. Conditional akaike information for mixed-effects models. Biometrika 92:351-370.

van Drunen WE, Dorken ME. 2012. Trade-offs between clonal and sexual reproduction in Sagittaria latifolia (Alismataceae) scale up to affect the fitness of entire clones. New Phytologist 196: 606-616.

van Kleunen M, Burczyk J. 2008. Selection on floral traits through male fertility in a natural plant population. Evolutionary Ecology 22: 39-54.

van Wilgen BW, Forsyth GG, de Klerk H, Das S, Khuluse S, Schmitz P. 2010. Fire management in Mediterranean-climate shrublands: a case study from the Cape fynbos, South Africa. Journal of Applied Ecology, 47: 631-638. 
Waelti MO, Page PA, Widmer A, Schiesti FP. 2009. How to be an attractive male: floral dimorphism and attractiveness to pollinators in a dioecious plant. BMC Evolutionary Biology 9: 190.

Welsford MR, Hobbhahn N, Midgley JJ, Johnson SD. 2016. Floral trait evolution associated with shifts between insect and wind pollination in the dioecious genus Leucadendron (Proteaceae). Evolution 70: 126-139.

Welsford, M. R., Midgley, J. J., \& Johnson, S. D. 2014. Experimental evaluation of insect pollination versus wind pollination in Leucadendron (Proteaceae). International journal of plant sciences 175: 296-306.

Wickham, H. 2016. ggplot2: Elegant Graphics for Data Analysis. Springer-Verlag New York, 2016.

Williams IJM. 1972. A revision of the genus Leucadendron (Proteaceae). Contrib. Bol. Herb 3: 1425.

Wright JW, Meagher TR. 2004. Selection on floral characters in natural Spanish populations of Silene latifolia. Journal of Evolutionary Biology 17: 382-395.

Zajitschek F, Connallon T. 2017. Partitioning of resources: the evolutionary genetics of sexual conflict over resource acquisition and allocation. Journal of Evolutionary Biology 30: 826838. 
894 All genotype and morphological trait data are available in Dryad at doi:10.5061/dryad.ngf1vhhst. 895 The new MEMMseedlings source code for dioecious species is available at 896 https://gitlab.paca.inrae.fr/pub/tonnabel mol ecol. The new version of spaMM is publicly available 897 in spaMM since version 2.6.0 at https://CRAN.R-project.org/package=spaMM.

\section{Author contributions}

900

IO, AM and AC designed the study, collected data, and mapped the plants; EKK produced the 901 mating model; FR implemented methods allowing fitting of sex-specific spatial random effects in spaMM; FR and AC designed the statistical procedures for measuring selection gradients in the presence of spatial autocorrelation; JT and AC performed the analyses; JT produced the genotyping dataset and drafted the manuscript; all authors discussed the results and edited the manuscript. 


\section{Figure captions}

Figure 1: Sexual dimorphism in canopy diameter in $\mathrm{cm}$ (A.) and leaf area in $\mathrm{cm}^{2}$ (B.) of the study population $L$. rubrum. Points are prediction from models accounting for the spatial distribution of traits within each sex and error bars are 95\% confidence intervals around mean predictions.

Figure 2: Dispersal kernels estimated under the Bayesian model for seed dispersal (A.) and for pollen dispersal (B.). Filled lines correspond to the posterior mean dispersal kernels obtained by averaging parameters of the concatenation of two Markov chains of 50,000 steps (burn-in phase of 10,000 steps). Grey lines illustrate the uncertainty around the averaged dispersal kernel and correspond to the kernels estimated on each iteration of the MCMC. Both dispersal kernels are represented within the minimal and maximal distances existing in our population between females and seedlings for seeds (A.) and between males and females for pollen (B.), i.e., respectively, the maximal female-seedling and male-female distances in our population. The extrapolation of dispersal kernels beyond these limits are not represented in the plots.

Figure 3: Spatial prediction of the relative effective fecundity in males (A.) and females (B.) as predicted by a generalized linear mixed-effect model, our full model, including all fixed effects (sex, canopy diameter, leaf area, plant density, and the interaction between each of the three latter variables and sex) as well as one spatially autocorrelated random effect for each sex. Relative fecundity represents our measure of relative effective fecundity estimated by our MEMMseedlings model, and circles represent the localization of individual plants.

Figure 4: Partial-dependence effect plots of leaf area (A.), canopy diameter (B.) and plant density (C.) on the relative effective fecundity as predicted by our full model. Points indicate observed trait values as well as the relative effective fecundity \pm SD stemming from MCMC estimations. Curves indicate model predictions computed as partial-dependence effects $\pm 95 \%$ confidence intervals. Relative fecundity designates our measure of relative effective fecundity.

Figure S1: Pictures of females and males Leucadendron rubrum plants (A.), and of male (B.) and female (C.) inflorescences magnification. Credit picture: Isabelle Olivieri.

Figure S2: Map of the study site showing distribution of females (red circles), males (blue triangles), juveniles (green crosses), non-genotyped females (red full circles) and non-genotyped males (blue full circles). One non-genotyped male that was located nearby the population is not represented in the map.

Figure S3: Results of a genetic analysis of the sampled individuals using the software STRUCTURE (Pritchard et al., 2000). Four types of samples were tested: juvenile plants of the third undetermined morphological group and, adult plants of L. salignum and of L. rubrum and juvenile plants of $L$. rubrum. Four genetic groups were selected by this analysis and are represented here by four different colors (i.e. blue, orange, pink and yellow). Individuals are displayed on two different panels (A. and B.) only for the sake of readability but all individuals belong to the same dataset described in Supplementary Methods S2.

Figure S4: Sexual dimorphism in plant height in $\mathrm{cm}$ (A.), in canopy first and second diameters in $\mathrm{cm}$ (B. \& C.), in leaf area in $\mathrm{cm}^{2}$ (D.), in leaf length in $\mathrm{cm}$ (E.) and in leaf width in $\mathrm{cm}$ (F.). Raw data have been jittered around their $\mathrm{x}$-axis value for visualization purposes and overlaid with both a violin plot and a boxplot using default settings in the R package ggplot2 version 3.1.0 (Wickham, 2016). 
Figure S5: Prediction of canopy diameter in males (A.) and females (B.) as predicted by a linear mixed-effect models including sex as a fixed effect as well as one spatially autocorrelated random effect for each sex.

Figure S6: Density for all individuals (A.), for males (B.) and for females (C.) as numbers of individuals in quadrats of $12 \times 12$ meters.

Figure S7: Comparisons of conditional likelihood between the model presented in the main text accounting for spatial structure of pollen dispersal (in green) and a model neglecting such a spatial structure of pollen dispersal (in brown) as a function of Bayesian iterations which were run for 50,000 steps. These simulations were run using uniform prior distributions with the following intervals [0.01,1.00], [0.01,1.00], [0.00,1.00], [1.00,100], [0.01,10], [1.00,30000], [0.01,1.10] for the parameters $m_{s}, m_{p}, v, \delta_{s}, b_{s}, \delta_{p}$ and $b_{p}$, except for the second model in which $\delta_{p}$ and $b_{p}$ were not modeled.

Figure S8: Replicate of Fig. 3 after the removal of individuals with effective fecundity greater than four standard errors (i.e. five females and one male). See Fig. 3 for legend details. Red crosses correspond to the locations of plants removed in the sensitivity analysis.

Figure S9: Spatial prediction of the number of empirically counted cones as predicted by a generalized linear mixed-effect model including all fixed effects (canopy diameter, leaf area, plant density) as well as one spatially autocorrelated random effect.

Figure S10: Replicate of Fig. 4 after the removal of individuals with effective fecundity greater than four standard errors (i.e. five females and one male). See Fig. 4 for legend details. 
A.

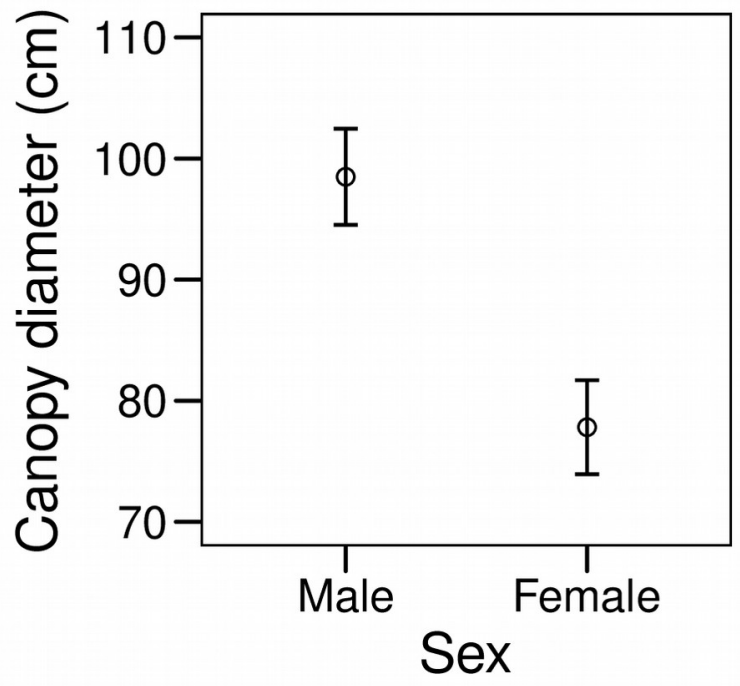

B.

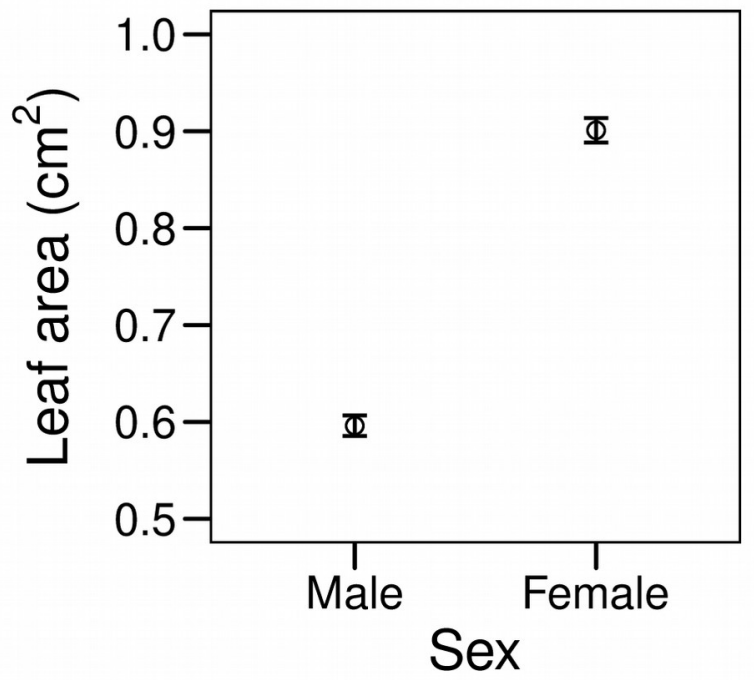


A.

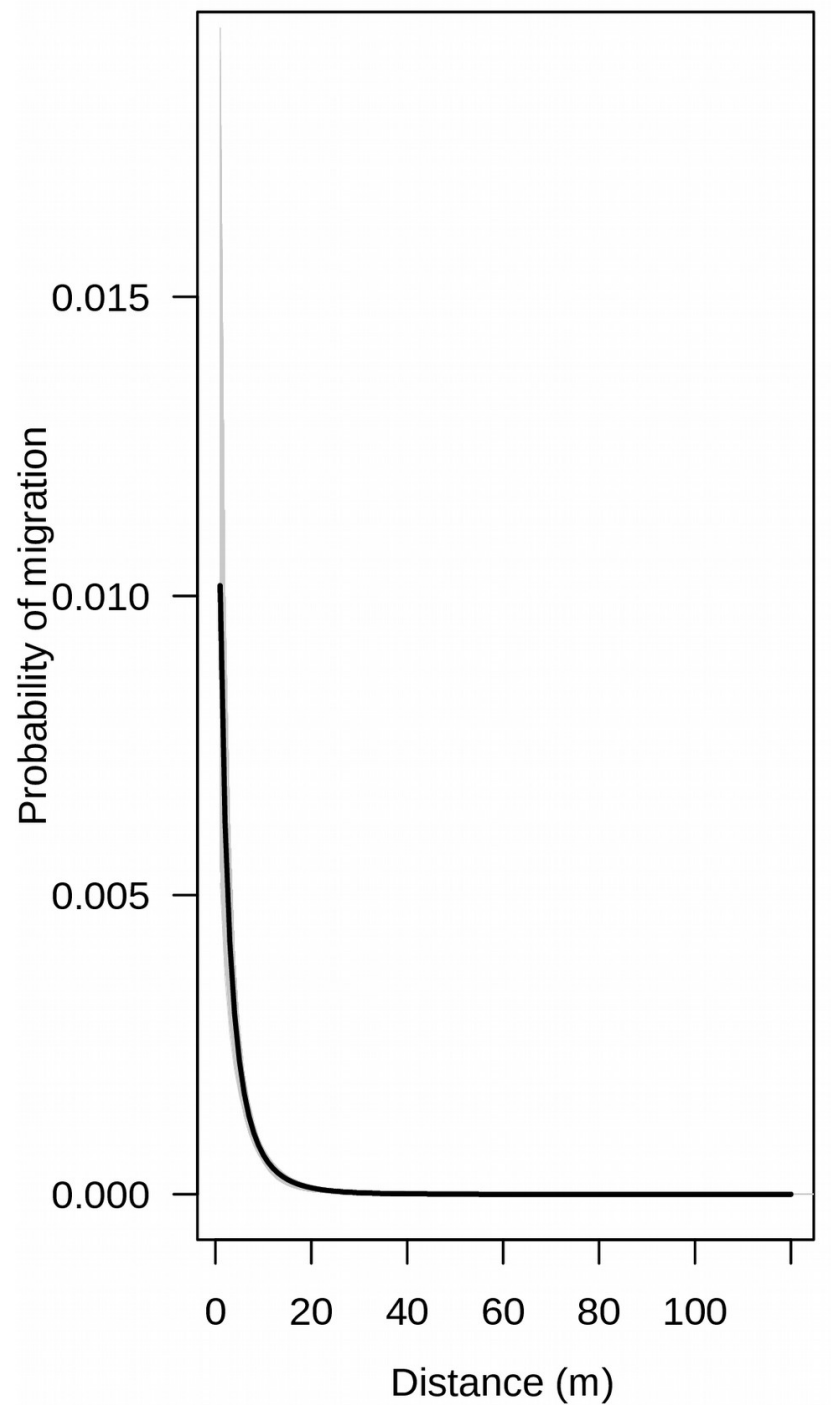

B.

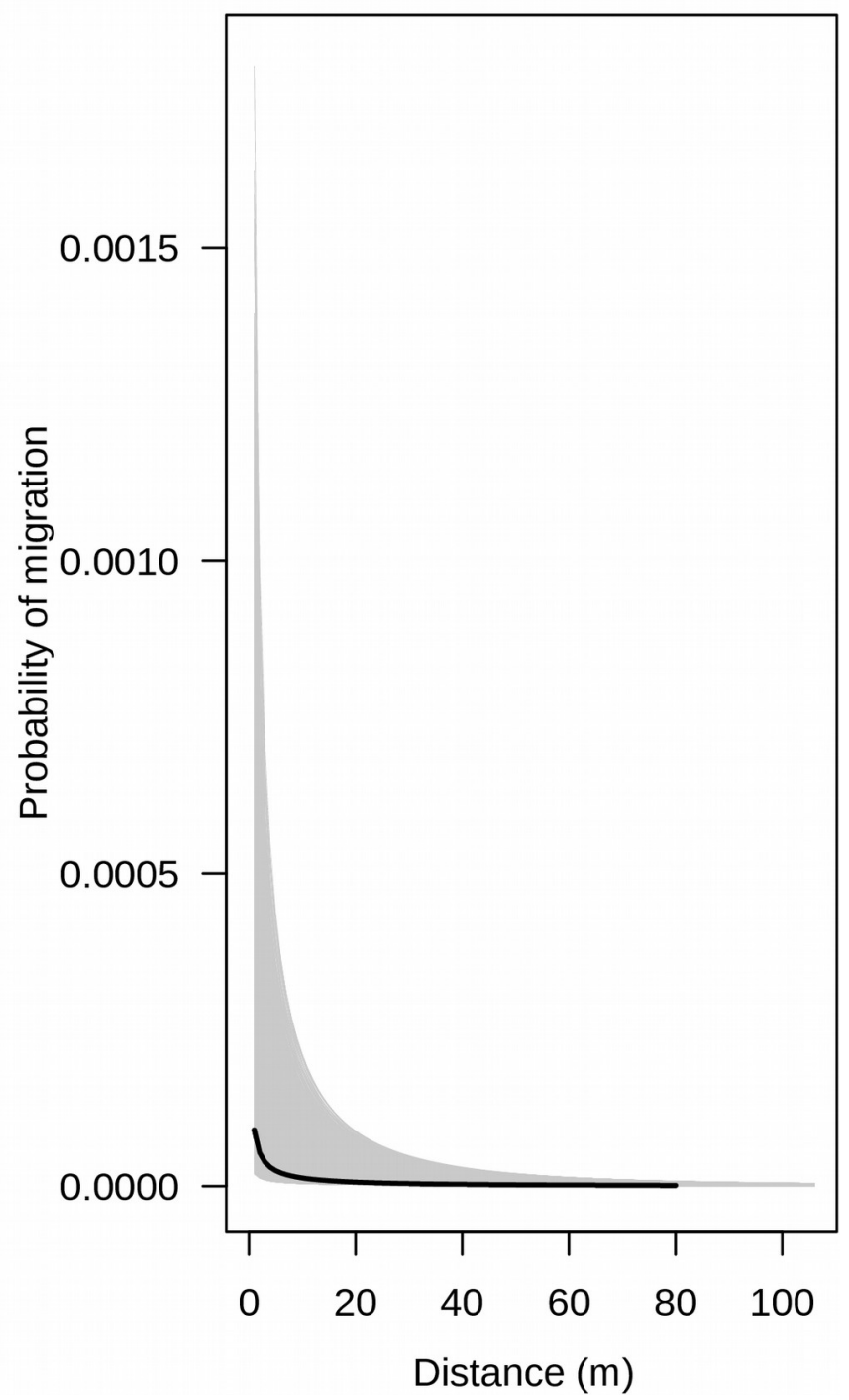

983 
A.

Males

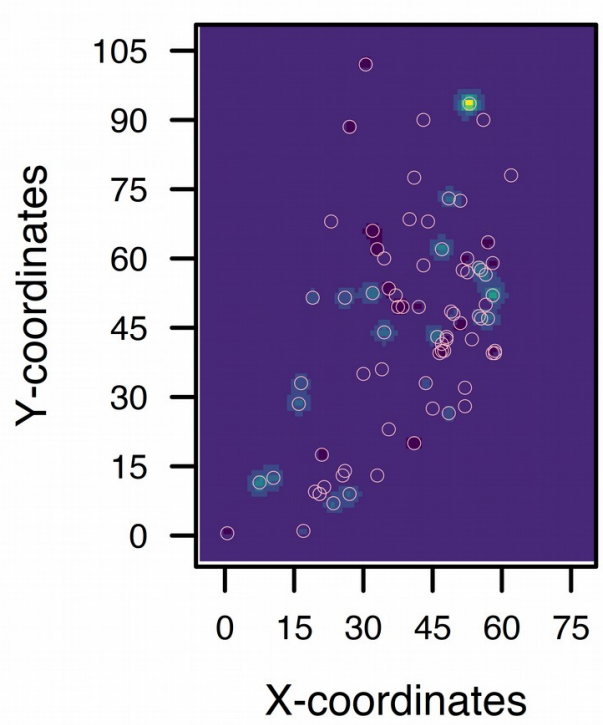

B.

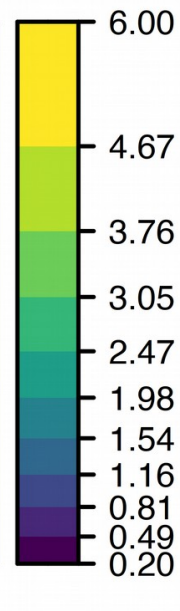

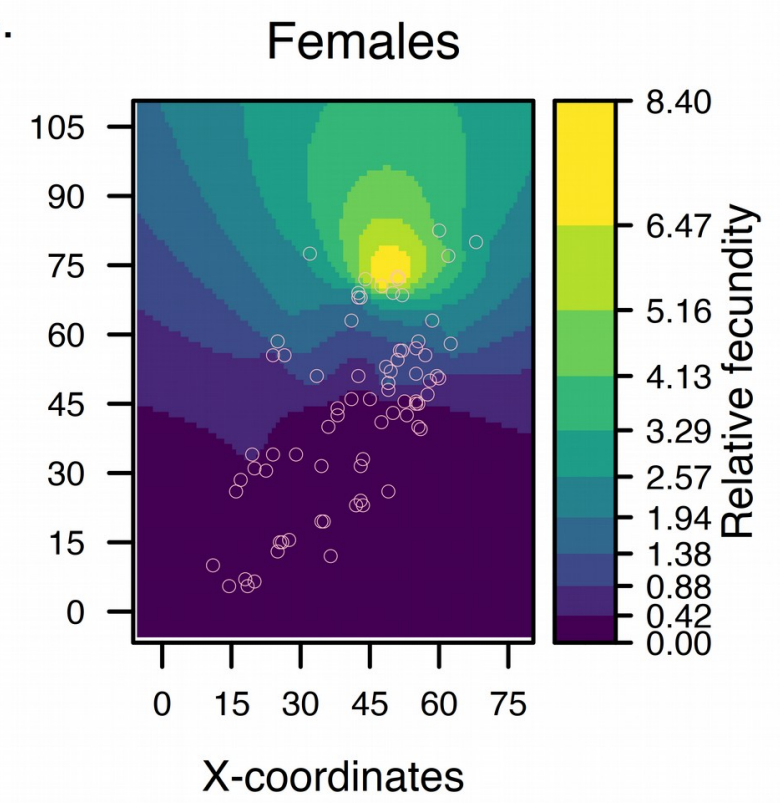



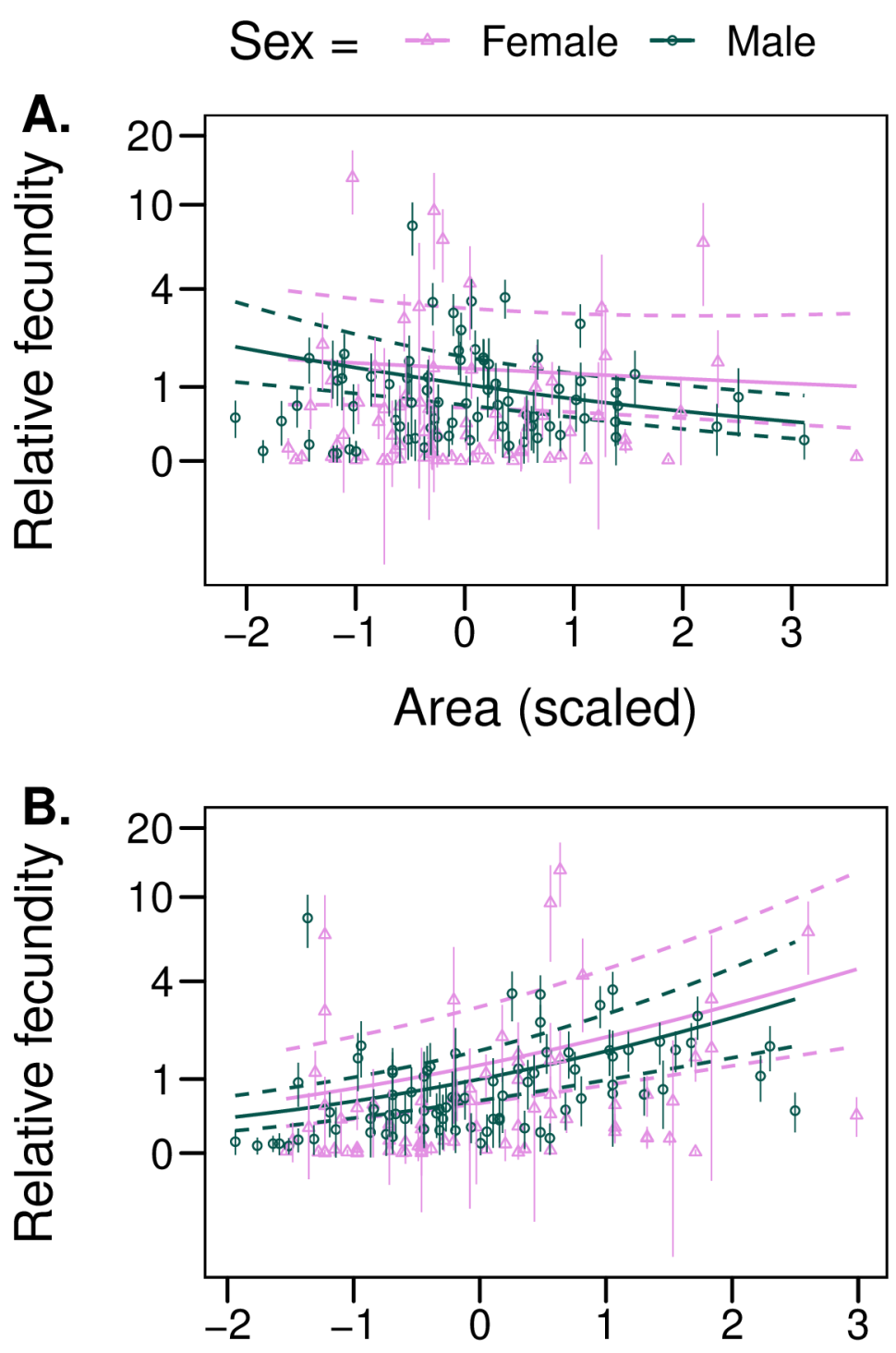

Diameter (scaled)

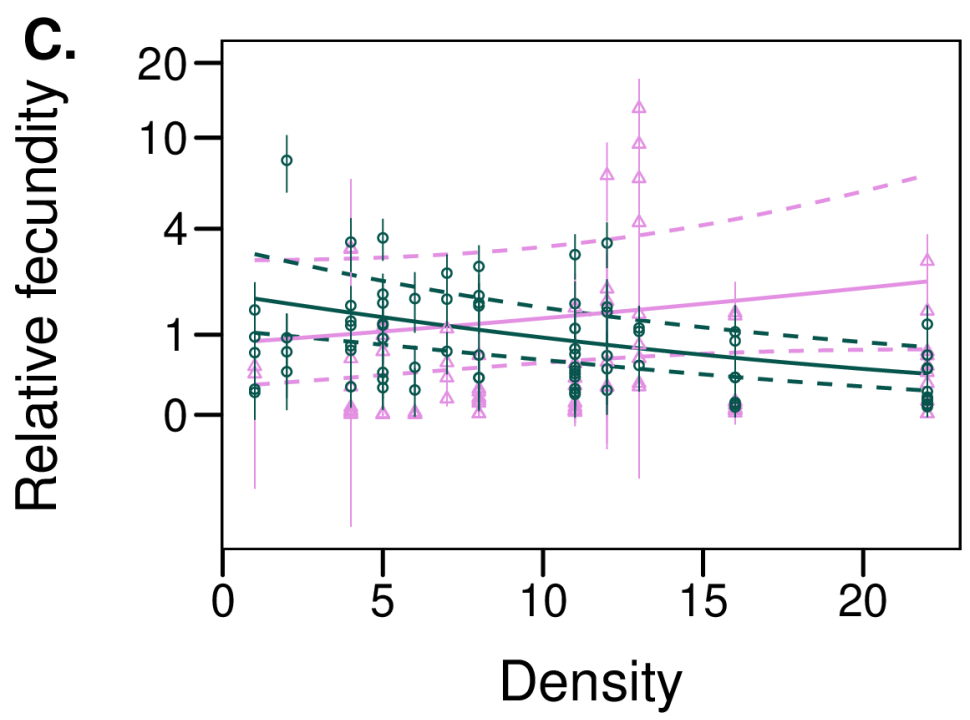

